British Journal of Psychiatry (1996), 169, 522-526

\title{
Correspondence
}

Contents: Steroid-induced prepartum psychosis/ Schizophrenia in twins/Screening for pathological gambling among substance misusers/Attention deficit disorder/In-patient psychotherapy/Antipsychotic drug-induced dysphoria/Inappropriate secretion of antidiuretic hormone and SSRIs/Seizure length during ECT/Suicide prevention versus alcohol promotion/Quaker belief and child psychiatry.

\section{Steroid-induced prepartum psychosis}

SIR: The possibility of puerperal psychosis having an onset before delivery has been suggested by Brockington et al (1990), but only a small number of cases have been reported in the literature. Glaze et al (1991) discussed the nature of the association between prepartum and postpartum psychoses and hypothesised that prepartum psychosis could occur as a result of sensitisation by earlier postpartum illness.

\section{Case report}

A 31-year-old woman presented at 36 weeks gestation with symptoms suggestive of hypomania. Two weeks previously she had been treated with prednisolone ( $20 \mathrm{mg} /$ day), for an infective exacerbation of asthma. The total dose prescribed was $60 \mathrm{mg}$. There was no relevant past psychiatric or family history.

The patient responded to haloperidol (dose range: 5-15 $\mathrm{mg}$ per day). Her symptoms gradually settled over the next month before being discharged home on no neuroleptic medication.

The patient remained stable until her subsequent (fourth) pregnancy. One week after delivery, she was admitted with a relapse of psychotic illness. During this admission the patient was treated with electroconvulsive therapy for escalating disturbance. She was discharged on lithium and haloperidol.

In this case the illness may have been precipitated by the use of steroids, predisposing the patient to later episodes of post-natal illness. Steroids are frequently prescribed in pregnancy-most commonly dexamethasone sulphate for premature rupture of membranes. If indeed steroid therapy has the potential to precipitate severe and recurring puerperal illness then prospective studies of pregnant women treated with dexamethasone would appear to be an appropriate focus of future research.

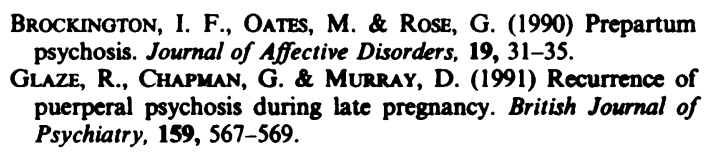

Barrow Hospital

I. JOHNSON

\section{Schizophrenia in twins}

SrR: Congratulations go to Kläning et al (1996) for their epidemiological demonstration that twins are more prone to schizophrenia than singletons. This is an important finding. It was one which we (Murray et al, 1985) predicted in an early formulation of the neurodevelopmental hypothesis of schizophrenia. If obstetric complications do constitute a risk factor for schizophrenia, and twins are known to be more prone to obstetric complications, then it had to follow that twins were at higher risk of schizophrenia. This is certainly the case with other neurodevelopmental disorders such as cerebral palsy and epilepsy.

An important implication which the authors do not discuss is that their finding effectively invalidates the classical twin method as a basis for estimating heritability in schizophrenia. A central assumption of the twin method is that the disorder under examination is no more common in twins than in singletons. That this might be the case was always hinted at by the apparently anomalous finding that concordance rates for schizophrenia between dizygotic (non identical) twins were consistently higher than those recorded amongst ordinary siblings despite the genetic relationship being the same in both cases. I hope that Kläning and colleagues can use their dataset to test further explanatory hypotheses about their findings. 